\title{
Efeitos da associação de tiletamina/zolazepam ou cetamina $S(+) /$ midazolam/tramadol para contenção química em bugios-ruivos (Allouatta guariba clamitans) $^{1}$
}

\author{
Pâmela Spolti², Aury N. de Moraes², Renato B. Tamanho², Martielo I. Gehrcke², \\ Júlio C. Souza Júnior ${ }^{3}$ e Nilson Oleskovicz ${ }^{2 *}$
}

\begin{abstract}
Spolti P., De Moraes A.N., Tamanho R.B., Gehrcke M.I., Souza Júnior J.C. \& Oleskovicz N. 2013 [Effects of tiletamine/zolazepam or S(+) ketamine/midazolam/ tramadol for chemical contention in red howler monkeys (Allouatta guariba clamitans).] Efeitos da associação de tiletamina/zolazepam ou cetamina $\mathrm{S}(+) /$ midazolam/tramadol para contenção química em bugios-ruivos (Allouatta guariba clamitans). Pesquisa Veterinária Brasileira 33(2):236-240. Departamento de Medicina Veterinária, Centro de Ciências Agroveterinárias, Universidade do Estado de Santa Catarina, Av. Luiz de Camões 2090, Lages, SC 88520-000, Brazil. E-mail: a2no@cav.udesc.br

Two protocols for chemical restraint in howler-redheads were evaluated. We used 12 healthy red howler monkeys, weighing $6.4 \pm 0.4 \mathrm{~kg}$, which were fasted and without water six and two hours, respectively. The animals were divided into two groups that received intramuscular injection: TZ $(n=6)$ which received tiletamine-zolazepam (Zoletil $\left.{ }^{\circledR}\right)$ at a dose of $3.6 \mathrm{mg} / \mathrm{kg}$ and CEMTRA $(\mathrm{n}=6)$ which received ketamine $\mathrm{S}(+)$, tramadol and midazolan (Cemtra ${ }^{\circledR}$, pilot batch 001/10, Ouro Fino Saúde Animal Ltda, Cravinhos/SP, Brazil, comprising $100 \mathrm{mg} / \mathrm{ml}$ of ketamine $\mathrm{S}(+)$, tramadol $20 \mathrm{mg} / \mathrm{ml}$ and $10 \mathrm{mg} / \mathrm{ml}$ of midazolam) in dose of $1 \mathrm{ml}$ of the association for each $10 \mathrm{~kg}$ of body weight, equivalent doses of $10 \mathrm{mg} / \mathrm{kg}$, $1 \mathrm{mg} / \mathrm{kg}$ and $2 \mathrm{mg} / \mathrm{kg}$, respectively. Prior to administration of drugs (M0) were evaluated: heart rate (HR), respiratory rate (RR), rectal temperature (RT), capillary refill time (CRT), systolic arterial pressure (SAP), hemoglobin oxygen saturation (SpO2), presence of salivation, degree of muscle relaxation and sedation, Bispectral index (BIS) and Signal Quality BIS (SQI), interdigital pinch response and latency times, ambulation and total recovery (TRT). The parameters were reassessed M5, M10, M20, M30, M40 and M50 (5, 10, 20, 30, 40 and 50 minutes after drug administration). In TZ animals were more responsive to the interdigital pinch over time. The animals of CEMTRA showed a higher degree of muscle relaxation and sedation. The RR of CEMTRA was lower after administration of treatment at all times in relation to M0. Among the groups RR of CEMTRA was lower compared to the TZ in M2 and M4. The total time of sedation and recovery was $48 \pm 4 \mathrm{~min}$ and $150.1 \pm 42.1$ for the CEMTRA and $38 \pm 7$ and $73.1 \pm 20.6$ for the TZ. We conclude that both formulations are safe for containing chemical howler, and the CEMTRA showed better sedation and muscle relaxant.
\end{abstract}

INDEX TERMS: Anesthetic association, anesthesia, monkeys, wild animal sedation, capture.

\footnotetext{
${ }^{1}$ Recebido em 25 de abril de 2012.

Aceito para publicação em 6 de setembro de 2012.

${ }^{2}$ Departamento de Medicina Veterinária, Centro de Ciências Agroveterinárias (CAV), Universidade do Estado de Santa Catarina (UDESC), Av. Luiz de Camões 2090, Lages, SC 88520-000, Brasil. *Autor para correspondência: a2no@cav.udesc.br

${ }^{3}$ Centro de Pesquisas Biológicas de Indaial e Observatório de Primatas - Projeto Bugio (Cepesbi), Rua Rio de Janeiro 401, Bairro dos Estados, Indaial, SC 89130-000, Brasil.
}

RESUMO.- Avaliaram-se dois protocolos para contenção química em bugios-ruivos. Para tal, foram utilizados 12 macacos bugios, hígidos, com peso médio de $6,4 \pm 0,4 \mathrm{~kg}$, os quais foram submetidos a jejum alimentar e hídrico de seis e duas horas, respectivamente. Os animais foram alocados em dois grupos que receberam injeção via intramuscular: TZ $(n=6)$, os quais receberam uma associação de tiletamina e zolazepam $\left(\right.$ Zoletil $^{\circledR}$ ) na dose de $3,6 \mathrm{mg} / \mathrm{kg}$ e CEMTRA 
$(\mathrm{n}=6)$, que receberam cetamina $\mathrm{S}(+)$, midazolam e tramadol (Cemtra $®$, lote piloto 001/10, Ouro Fino Saúde Animal Ltda., Cravinhos, SP-Brasil, constituído por $100 \mathrm{mg} / \mathrm{ml}$ de cetamina $\mathrm{S}+, 20 \mathrm{mg} / \mathrm{ml}$ de tramadol e $10 \mathrm{mg} / \mathrm{ml}$ de midazolam) na dose de $1 \mathrm{ml}$ da associação para cada $10 \mathrm{~kg}$ de peso corporal, correspondendo às doses de $10 \mathrm{mg} / \mathrm{kg}, 1 \mathrm{mg} / \mathrm{kg}$ e $2 \mathrm{mg} / \mathrm{kg}$, respectivamente. Anteriormente a administração dos fármacos (M0) foram avaliadas: frequência cardíaca (FC) e respiratória (f), temperatura retal (TR), tempo de preenchimento capilar (TPC), pressão arterial sistólica (PAS), saturação de oxigênio na hemoglobina $\left(\mathrm{SpO}_{2}\right)$, presença de salivação, grau de miorrelaxamento e sedação, índice Bispectral (BIS) e Sinal de Qualidade do BIS (SQI), resposta ao pinçamento interdigital e tempos de latência, deambulação e de recuperação total (TRT). Os parâmetros foram reavaliados em M5, M10, M20, M30, M40 e M50 (5, $10,20,30,40$ e 50 minutos após a administração dos fármacos). No TZ os animais foram mais responsivos ao pinçamento interdigital ao longo dos tempos. Os animais do CEMTRA apresentaram maior grau de miorrelaxamento e de sedação. A $f$ do CEMTRA foi menor após a administração do tratamento em todos os momentos em relação ao M0. Entre grupos a $f$ do CEMTRA foi menor em relação ao TZ em M2 e M4. Os tempos totais de sedação e de recuperação foram de $48 \pm 4$ e 150,1 $\pm 42,1$ min para o CEMTRA e de $38 \pm 7$ e $73,1 \pm 20,6$ para o TZ. Conclui-se que ambas as formulações são seguras para contenção química de bugios, sendo que o CEMTRA apresentou melhor sedação e miorrelaxamento.

TERMOS DE INDEXAÇÃO: Associação anestésica, anestesia, macacos, sedação de animais selvagens, captura.

\section{INTRODUÇÃO}

A contenção química de animais silvestres, muitas vezes, é fundamental para que os procedimentos da clínica veterinária e colheita de material para exames laboratoriais e pesquisas possam ser realizados. Ainda, frequentemente, é o único meio para a captura, transporte e tratamento dos animais. Assim, é reconhecidamente uma prática comum no manejo de animais silvestres e um importante componente de pesquisa (Pitt et al. 2006).

Durante a captura, o estresse é um fator predominante, frequente e limitante no manejo de animais selvagens, visto que um estímulo prolongado de estresse pode até levar o animal à morte. Assim, é importante que se utilizem fármacos que possam minimizar o estresse na captura (Acco et al. 1999).

Entretanto, os fármacos disponíveis para uso em animais selvagens muitas vezes têm seus efeitos e doses extrapolados das espécies domésticas, podendo apresentar efeitos inesperados. 0 uso da associação tiletamina-zolazepam (TZ) em animais selvagens e exóticos tem sido amplo por requerer pequeno volume de injeção, ter boa absorção por via intramuscular, possuir ampla margem de segurança em animais hígidos e permitir desde imobilização até anestesia (Lin 1993, Pitt et al. 2006). Porém, o uso desta associação ainda é controverso em algumas espécies por falta de estudos, apesar das recomendações de fabricantes para espécies do mesmo gênero.
A cetamina é amplamente utilizada para imobilização de animais selvagens, em virtude de sua elevada dose letal (DL 50) que permite seu uso sem o conhecimento do peso exato do animal, e da boa absorção por via intramuscular, o que permite a administração por meio de dardos (Diniz 1996). Entretanto, deve ser usada em associação com outros fármacos, como os benzodiazepínicos, pois pode causar estímulo cardiovascular, catalepsia e recuperação agitada (Haskins et al. 1985).

Embora em humanos o isômero levógiro $\mathrm{S}(+)$ da cetamina apresente maior potência e menores efeitos colaterais (Lauretti et al. 2000), em cães (Duque et al. 2008) e em macacos do gênero Callithrix (Furtado et al. 2010) não se observaram diferenças da forma racêmica.

0 tramadol é um opioide considerado atípico, pois possui mecanismo de ação analgésica não opioides como liberação e recaptação de serotonina e noradrenalina, assim, possui um excelente efeito analgésico, sem a ocorrência de efeitos indesejáveis dos demais opioides, como depressão cardiorrespiratória e hipomotilidade gastrointestinal (Lee et al. 1993). Segundo Dayer et al. (1997) após administração por via intramuscular em humanos, o efeito analgésico do tramadol pode se estender por 6 a 8 horas. Todavia, não se encontram dados na literatura relacionados com a utilização de tramadol em bugios, nem tampouco em outros primatas não-humanos.

Diante da necessidade de se utilizar contenção química para manejo de animais silvestres e da falta de estudos em algumas espécies o objetivo deste trabalho foi avaliar os efeitos clínicos da administração da associação de cetamina S+/midazolam/tramadol e compará-la a associação comumente utilizada de tiletamina/zolazepam em bugios ruivos (Allouatta guariba clamitans), visto que não existem fármacos aprovados para uso nesta espécie.

\section{MATERIAL E MÉTODOS}

Foram utilizados 12 macacos bugios (Alouatta guariba clamitans), com peso médio de $6,4 \pm 0,4 \mathrm{~kg}$, machos, com idade estimada de 5 a 10 anos, provenientes de um centro de conservação e pesquisas biológicas, sendo o estudo aprovado pelo comitê de ética e bem estar animal da instituição de origem (Protocolo 1.07/09.). Os animais tiveram sua higidez atestada após exame físico e perfil bioquímico hepático e renal. Previamente ao estudo, os animais foram submetidos a jejum alimentar por um período de seis horas e jejum hídrico de duas horas.

Os animais foram alocados aleatoriamente em dois grupos que receberam os fármacos por via intramuscular: $\mathrm{TZ}(\mathrm{n}=6)$, os quais receberam a associação de tiletamina e zolazepam (Zoletil ${ }^{\circledR}$ ) na dose de $3,6 \mathrm{mg} / \mathrm{kg}$, que é a preconizada pelo fabricante para espécies do mesmo gênero, e grupo CEMTRA $(n=6)$, os quais receberam uma associação de cetamina $\mathrm{S}(+)$, midazolam e tramadol (Cemtra ${ }^{\circledR}$, lote piloto 001/10, Ouro Fino Saúde Animal Ltda, Cravinhos, SP, constituído por $100 \mathrm{mg} / \mathrm{ml}$ de cetamina $\mathrm{S}+, 20 \mathrm{mg} /$ $\mathrm{ml}$ de tramadol e $10 \mathrm{mg} / \mathrm{ml}$ de midazolam) na dose de $1 \mathrm{ml}$ da associação para cada $10 \mathrm{~kg}$ de peso corporal, correspondendo às doses de $10 \mathrm{mg} / \mathrm{kg}, 1 \mathrm{mg} / \mathrm{kg}$ e $2 \mathrm{mg} / \mathrm{kg}$. A distribuição dos animais nos respectivos grupos, bem como a ordem de administração dos fármacos foi estabelecida através de sorteio, e as avaliações realizadas por um único pesquisador cego aos tratamentos.

Após captura, era realizada contenção física manual, por um profissional experiente, o qual posicionava os animais sobre uma 
Quadro 1. Valores médios e desvios-padrão de frequência cardíaca (FC), pressão arterial sistólica (PAS), frequência respiratória $(f)$, temperatura retal (TR), saturação parcial de oxigênio $\left(\mathrm{SPO}_{2}\right)$, índice bispectral (BIS) e sinal de qualidade do BIS (SQI) após a administração de cetamina/midazolam/ tramadol (CEMTRA) ou tiletamina/zolazepam (TZ), em bugios-ruivos (Allouatta guariba clamitans)

\begin{tabular}{ccccccccc}
\hline Variável & Grupos & M0 & M5 & M10 & M20 & M30 & M40 & M50 \\
\hline FC (bat/min) & CEMTRA & $193,3 \pm 36,1$ & $190,3 \pm 21,3$ & $167 \pm 25,0$ & $162 \pm 33,9$ & $158,6 \pm 34,1$ & $164,3 \pm 26,1$ & $173,2 \pm 44,1$ \\
& TZ & $191,3 \pm 47,2$ & $193,3 \pm 16,3$ & $168,1 \pm 30,6$ & $171,6 \pm 39,1$ & $170 \pm 36$ & $178,4 \pm 39,3$ & $190 \pm 0$ \\
PANI (mmHg) & CEMTRA & $128 \pm 13$ & $110 \pm 14$ & $117 \pm 25$ & $117 \pm 27$ & $128 \pm 30$ & $125 \pm 27$ & $123 \pm 30$ \\
& TZ & $121 \pm 24$ & $122 \pm 21$ & $126 \pm 25$ & $122 \pm 22$ & $117 \pm 14$ & $117 \pm 15$ & $116 \pm 0$ \\
F (mov/min) & CEMTRA & $21,6 \pm 2,6$ & $15,3 \pm 3,9 \mathrm{~A}$ & $17,3 \pm 2,0 \mathrm{Ab}$ & $15,3 \pm 1,6 \mathrm{~A}$ & $16,6 \pm 3,0 \mathrm{Ab}$ & $16,6 \pm 3,0 \mathrm{~A}$ & $14,4 \pm 3,2 \mathrm{a}$ \\
& TZ & $22,6 \pm 3,2$ & $18,6 \pm 3,2$ & $20,6 \pm 3,0 \mathrm{a}$ & $20 \pm 5,0$ & $21,3 \pm 4,1 \mathrm{a}$ & $18 \pm 1,7$ & $12 \pm 0$ \\
$\mathrm{TR}\left({ }^{\circ} \mathrm{C}\right)$ & CEMTRA & $37,8 \pm 1,7$ & $38,4 \pm 1,6$ & $38,4 \pm 1,3$ & $38,4 \pm 0,8$ & $38,0 \pm 0,9$ & $37,8 \pm 0,7$ & $37,5 \pm 0,6$ \\
& TZ & $38,9 \pm 0,7$ & $39,1 \pm 0,4$ & $38,8 \pm 0,4$ & $38,5 \pm 0,4$ & $38,4 \pm 0,5$ & $38,2 \pm 0,4$ & $37,4 \pm 0$ \\
$\mathrm{SPO}_{2}(\%)$ & CEMTRA & $95,6 \pm 2,0$ & $95 \pm 1,7$ & $96,6 \pm 1,6$ & $95,4 \pm 2,1$ & $98 \pm 1,2$ & $94,4 \pm 1,0$ & $96,7 \pm 1,7$ \\
\multirow{2}{*}{ BIS } & TZ & $96 \pm 0,6$ & $96 \pm 1,4$ & $98,4 \pm 0,4$ & $97,2 \pm 0,7$ & $96 \pm 2,7$ & $96,7 \pm 2,1$ & $98 \pm 0$ \\
& CEMTRA & - & - & $75,2 \pm 9,9$ & $70,5 \pm 15,7$ & $81,4 \pm 4,6$ & $81,25 \pm 4,3$ & $79 \pm 7,2$ \\
SQI & TZ & - & $82 \pm 0$ & $76,6 \pm 5,8$ & $77 \pm 6$ & $80,5 \pm 9,1$ & $87 \pm 0$ & - \\
& CEMTRA & - & - & $75 \pm 10$ & $68,7 \pm 8,4$ & $81,4 \pm 13,5$ & $73,7 \pm 6,6$ & $69,6 \pm 16,1$ \\
& TZ & - & $64 \pm 0$ & $65 \pm 19,4$ & $82,3 \pm 20,1$ & $81 \pm 5,6$ & $83 \pm 0$ & -
\end{tabular}

Letras maiúsculas na mesma linha entre as colunas significam diferença de M0.

Letras minúsculas diferentes na mesma coluna entre linhas significam diferença entre grupos após $(\mathrm{p} \leq 0,05)$.

mesa, com os pés apoiados, em posição ereta, com os braços flexionados por trás do animal, evitando acidentes e maior estresse aos mesmos. Após contenção dos animais, eram aferidos os parâmetros basais (M0), referentes à: frequência cardíaca (FC), frequência respiratória $(f)$, tempo de preenchimento capilar (TPC), coloração das mucosas, temperatura retal (TR), pressão arterial sistólica (PAS) com a utilização do Doppler posicionado sobre a artéria braquial; e saturação parcial de oxigênio $\left(\mathrm{SpO}_{2}\right)$ por meio de oxímetro de pulso, com sensor posicionado no dígito do animal.

Aferidos os parâmetros basais, foi realizada a administração dos tratamentos, na musculatura do membro pélvico direito. Em ato contínuo, os animais foram alocados em gaiolas individuais, onde era observado o período de latência (em minutos), considerando este, o momento no qual o animal apresentava-se em decúbito lateral. Os parâmetros avaliados no basal (M0) foram novamente avaliados em: M5, M10, M20, M30, M40 e M50, correspondentes a $5,10,20,30,40$ e 50 minutos, respectivamente, após a aplicação.

Além dos parâmetros avaliados em M0, foram também avaliados: o índice bispectral (BIS) e sinal de qualidade do BIS (SQI), conectando-se o sensor, com dois eletrodos frontais e um temporal; grau de miorrelaxamento $(0=$ sem relaxamento, $1=$ leve, $2=$ moderado, 3 = ótimo), pela resistência à movimentação dos membros inferiores, superiores e pescoço; reflexo podal e caudal (presente ou ausente), por meio da tração dos mesmos e verificação do relaxamento da cauda; resposta ao pinçamento interdigital (presente ou ausente), realizado com o auxílio de uma pinça hemostática, a qual era pressionada até a primeira cremalheira no espaço interdigital; qualidade de sedação $(0=$ sem sedação, $1=$ levanta-se quando estimulado, 2 = reage a estímulos, mas permanece deitado, 3 = não reage a estímulos); qualidade de recuperação ( $0=$ ruim, 1= boa, 2= ótima); tempo de recuperação (em minutos); tempo para deambulação, considerando o momento a partir do qual o animal levantava se, mas ainda não conseguia permanecer em posição bipedal, e tempo de recuperação total, considerando este o momento no qual o animal permanecia em estação sem dificuldades. As avaliações dos parâmetros subjetivos foram realizadas e avaliadas sempre pelo mesmo examinador cego aos tratamentos, conforme descrito por Chagas et al. (2010).

Todos os dados paramétricos foram analisados pelo programa computacional Sigma Stat for Windows (2009), aplicando se o teste-t entre os grupos. Entre os tempos dentro de cada grupo, utilizou se o teste de Análise de variância de uma única via com repetições múltiplas (ANOVA), e as diferenças encontradas foram analisadas pelo teste de Student-Newman-Keuls $(\mathrm{p} \leq 0,05)$.

\section{RESULTADOS E DISCUSSÃO}

Não se observaram diferenças significativas na FC, PAS, $\mathrm{SpO}_{2}$, TR e TPC tanto entre grupos como dentre grupos (Quadro 1). Entretanto, a $f$ no grupo TZ foi maior, diferindo significativamente do CEMTRA em M10 e M30, possivelmente devido à hipertonicidade muscular e ao aumento da $\mathrm{PaCO}_{2}$ desencadeada pela tiletamina (Lin 1996), sendo esse resultado encontrado em outras espécies tratadas com a associação TZ (Lin et al. 1993). Porém, como essa variável não foi mensurada em nosso estudo não podemos afirmar esta causa. Em contrapartida, o os animais do grupo TZ apresentavam menor grau de sedação, o que pode resultar em uma maior $f$.

No grupo CEMTRA houve diminuição da $f$ em todos os momentos após aplicação do tratamento sendo diferentes de M0. Hanskins et al. (1985) demonstraram diminuição da $f$ e do volume minuto durante aproximadamente $15 \mathrm{mi}-$ nutos em cães anestesiados com cetamina . Furtado et al. (2010), encontraram os mesmos resultados em macacos do gênero Callihtrix, verificando diminuição da $f$ com doses similares de cetamina e midazolam. Cabe ressaltar que, no presente estudo, a avaliação dos parâmetros cessava após o fim da sedação, assim alguns animais nos últimos momentos não foram avaliados para evitar maior estresse dos mesmos. Ainda, embora os animais fossem condicionados a este manejo no criatório, é possível que a reposta de estresse possa alterar os valores basais (Quadro 1), que após a sedação naturalmente diminuiriam (Fowler 1986, Acco et al. 1999).

Não foram observadas diferenças significativas no tempo de latência de ambos os grupos (Quadro 2). Em relação à qualidade de sedação, o grupo CEMTRA demonstrou ser superior ao grupo TZ durante todos os momentos, sendo que até M10, apresentou 100\% dos animais com escore máximo de qualidade de sedação (Quadro 3). Ainda, ob- 
Quadro 2. Valores médios e desvios-padrão de tempo (minutos) de latência, tempo total de sedação, tempo de deambulação e tempo total de recuperação após a administração de cetamina/midazolam/tramadol (CEMTRA) ou tiletamina/zolazepam (TZ), em bugios-ruivos (Allouatta guariba clamitans)

\begin{tabular}{|c|c|c|}
\hline Variável & CEMTRA & $\mathrm{TZ}$ \\
\hline Tempo de latência (min) & $2,8 \pm 0,7$ & $3,1 \pm 0,7$ \\
\hline Tempo total de sedação (min) & $48 \pm 4 *$ & $38 \pm 7$ \\
\hline Tempo de deambulação (min) & $68 \pm 17$ & $56 \pm 14$ \\
\hline Tempo de recuperação total (min) & $150 \pm 42 *$ & $73 \pm 20$ \\
\hline
\end{tabular}

Quadro 3. Frequência de animais que apresentaram ótimo grau de sedação, relaxamento muscular intenso e ausência de resposta ao pinçamento interdigital após a administração de cetamina/midazolam/tramadol (CEMTRA) ou tiletamina/zolazepam (TZ), em bugios-ruivos (Allouatta guariba clamitans)

M0 M5 M10 M20 M30 M40 M50

\begin{tabular}{|c|c|c|c|c|c|c|}
\hline Grau de sedação ótimo & $\begin{array}{l}\text { CEMTRA } \\
\text { TZ }\end{array}$ & $\begin{array}{ll}- & 6 / 6 \\
- & 4 / 6\end{array}$ & $\begin{array}{l}6 / 6 \\
4 / 6\end{array}$ & $\begin{array}{l}5 / 6 \\
2 / 6\end{array}$ & $\begin{array}{l}4 / 6 \\
2 / 6\end{array}$ & $\begin{array}{l}3 / 6 \\
0 / 6\end{array}$ \\
\hline $\begin{array}{r}\text { Relaxa } \\
\text { tens }\end{array}$ & $\begin{array}{c}\text { CEMTRA } \\
\text { TZ }\end{array}$ & $\begin{array}{ll}- & 6 / 6 \\
- & 3 / 6\end{array}$ & $\begin{array}{l}5 / 6 \\
3 / 6\end{array}$ & $\begin{array}{l}4 / 6 \\
2 / 6\end{array}$ & $\begin{array}{l}3 / 6 \\
1 / 6\end{array}$ & $\begin{array}{l}2 / 6 \\
0 / 6\end{array}$ \\
\hline $\begin{array}{l}\text { Ausência de resposta pir } \\
\text { çamento }\end{array}$ & $\begin{array}{c}\text { CEMTRA } \\
\text { TZ }\end{array}$ & $\begin{array}{l}-5 / 6 \\
-\quad 5 / 6\end{array}$ & $\begin{array}{l}5 / 6 \\
4 / 6\end{array}$ & $\begin{array}{l}4 / 6 \\
2 / 6\end{array}$ & $\begin{array}{l}3 / 6 \\
1 / 6\end{array}$ & $\begin{array}{l}2 / 6 \\
0 / 6\end{array}$ \\
\hline ssên & $\begin{array}{c}\text { CEMTRA } \\
\text { TZ }\end{array}$ & $\begin{array}{l}6 / 6 \\
6 / 6\end{array}$ & $\begin{array}{l}5 / 6 \\
5 / 6\end{array}$ & $\begin{array}{l}3 / 6 \\
3 / 6\end{array}$ & $\begin{array}{l}3 / 6 \\
0 / 6\end{array}$ & $\begin{array}{l}3 / 6 \\
0 / 6\end{array}$ \\
\hline
\end{tabular}

servou se em M5 que em ambos os grupos 83,3\% dos animais não responderam ao pinçamento interdigital. Porém, no decorrer do estudo, a ausência do reflexo ao pinçamento ocorreu em um maior número de animais do CEMTRA e perdurou por mais tempo (Quadro 3). Estes dados corroboram Chagas et al. (2010), que obtiveram um adequado grau de sedação em animais da mesma espécie aonde por meio de extrapolação alométrica os autores chegaram a doses médias de cetamina e midazolam de $9,5 \mathrm{mg} / \mathrm{kg}$ e $0,92 \mathrm{mg} /$ kg respectivamente. É provável que a dose utilizada da associação TZ seja baixa para captura e contenção química, pois além de não haver dados específicos do uso da associação para esta espécie, em um estudo com bugios da espécie seniculus, cujo fabricante recomenda a dose de $3,6 \mathrm{mg} / \mathrm{kg}$, os autores concluíram que a dose para adequada imobilização química desta espécie seria de $22,5 \mathrm{mg} / \mathrm{kg}$, pois produz o miorrelaxamento necessário para que a cauda preênsil perca sua capacidade e o animal posa ser capturado (Agoramoorthy \& Rudran 1994). Somando se a isto, o grupo CEMTRA envolve o uso de tramadol, que em humanos e cães apresenta potencialização da sedação quando associado a outros agentes sedativos (Monteiro et al. 2009). Porém, nesta espécie não se encontram relatos referentes ao uso deste fármaco e seus efeitos.

Em relação ao BIS e SQI, não se observaram diferenças tanto entre grupos como dentre grupos (Quadro 1), permanecendo com valores acima dos considerados indicadores de sedação em humanos e pequenos animais. No entanto, os animais do grupo CEMTRA apresentaram, visualmente, ótimo grau de sedação sendo superior ao grupo TZ. (Quadro 3). Isto deve se ao fato de que a utilização de diferentes fármacos pode alterar os valores de BIS. Estudos demonstraram que a utilização de cetamina, devido aos seus mecanismos dissociativos interfere nos sinais elétricos do sistema nervoso central, podendo fornecer valores de BIS alterados e acima dos valores esperados para sedação profunda e anestesia em pacientes humanos (Friedberg 1999, Hans et al. 2005). Nesta espécie não se dispõe de dados a respeito do BIS para avaliação de sedação, porém sabe se que os anestésicos dissociativos podem alterar os valores como citado acima, devendo se interpretar os dados deste estudo com cautela.

O CEMTRA apresentou ótimo grau de miorrelaxamento em todos os animais até M10 (Quadro 3), enquanto que neste mesmo momento no TZ, $16,66 \%$ dos animais (1/6) apresentaram qualidade de miorrelaxamento ruim, $33,33 \%$ dos animais $(2 / 6)$ apresentaram miorrelaxamento considerado bom e $50 \%(3 / 6)$ considerado ótimo. No CEMTRA, a partir de M20, foi observado um maior número de animais com escore de miorrelaxamento ótimo em todos os momentos, quando comparado ao TZ. Pode se observar no CEMTRA, a ausência do reflexo caudal, até 30 minutos após a administração dos fármacos em 83,3 \% (5/6) dos animais, quando comparado ao TZ, que apresentou ausência deste reflexo em 33,3\% (2/6) dos animais. Ainda no grupo $\mathrm{TZ}$, após M30,100\% dos animais apresentaram presença do reflexo caudal. Em M5 o CEMTRA apresentou ausência de reflexo podal em $100 \%$ dos animais, diferindo do TZ, o qual apresentou em M5 ausência em 66,6\% e presença em $33,3 \%$ dos animais. Em M10 o reflexo esteve ausente em 83,3\% no CEMTRA e 50\% no TZ. Os dois grupos apresentaram escores idênticos (50\%) em M20. Nos demais momentos, o CEMTRA apresentou ausência do reflexo podal por mais tempo e também em um maior número de animais, quando comparado ao TZ .

Neste sentido, quando se propõem protocolos para sedação de animais desta espécie, um dos principais indicativos de uma boa sedação é o reflexo caudal, pois na presença deste, o animal mesmo sedado pode prender a cauda em estruturas fixas, como galhos, ficando inacessível (Glander et al. 1991). No grupo CEMTRA pode-se observar melhor miorrelaxamento e perda de reflexo caudal e podal quando comparado ao grupo TZ. Pode se atribuir isto ao efeito miorrelaxante do midazolam e pela dose utilizada, pois resultados semelhantes foram encontrados por Chagas et al. (2010) e Furtado et al. (2010) nas mesmas dosagens. Ainda, a dose utilizada no grupo TZ pode ter sido insuficiente para produzir maior grau de miorrelaxamento visto que a dose foi extrapolada a partir do que recomenda a bula para espécies similares do mesmo gênero. Corroborando Agoramoorthy \& Rudran (1994) que verificaram a necessidade de doses maiores que as recomendadas da associação TZ para contenção de espécies do mesmo gênero estudado.

Um dos grandes problemas na contenção de animais silvestres é o fato de que os protocolos utilizados apresentam pobre analgesia, pois não se encontram estudos com a utilização de analgésicos opioides. Levando-se em consideração que procedimentos como limpeza de feridas, suturas e 
curativos são frequentes nestas espécies, a presença de um agente analgésico torna-se fundamental (Pitt et al. 2006). Entretanto, neste estudo não se utilizou métodos para se determinar o grau de analgesia do protocolo, pois o reflexo ao pinçamento discutido anteriormente reflete a sedação e os reflexos motores e não a qualidade analgésica (Monteiro et al. 2009). Embora encontre se estudos em humanos (Ayoglu et al. 2010) e suínos (Ajadi et al. 2009) que comprovem a associação benéfica da cetamina com o tramadol para aumentar o grau de analgesia dos pacientes, ainda são necessários mais estudos com relação à utilização e dosagem do tramadol em espécies selvagens.

Em relação ao tempo total de sedação $(38 \pm 7$ min no TZ e 48 \pm 4 min no CEMTRA) e tempo de recuperação total $(150 \pm 42 \mathrm{~min}$ no CEMTRA e de $73 \pm 20 \mathrm{~min}$ no TZ), observou se maiores valores no grupo CEMTRA, diferindo estatisticamente do grupo TZ. Os tempos totais de sedação e de recuperação total no grupo TZ foram inferiores aos encontrados por Agoramoorthy \& Rudran (1994) em espécies do mesmo gênero deste estudo, entretanto os autores utilizaram doses maiores da associação. Já os valores encontrados no grupo CEMTRA para tempo total de sedação corroboraram Chagas et al. (2010) que utilizaram as mesmas doses de cetamina e midazolam, porém, os autores não avaliaram o tempo total de recuperação. Os maiores tempos de sedação e recuperação encontrados no CEMTRA comparados ao grupo $\mathrm{TZ}$ referem se às doses empregadas, que também resultaram em um grau de sedação inferior no TZ.

O tempo para deambulação não diferiu entre os grupos. A qualidade de recuperação foi classificada como boa em $83,3 \%$ dos animais e ruim em apenas $16,6 \%$ de ambos os grupos. Chagas et al. (2010) também encontraram boa qualidade de recuperação em animais da mesma espécie com a associação cetamina/midazolam.

\section{CONCLUSÃO}

Conclui se que ambas as associações, tiletamina-zolazepam ou cetamina, midazolam e tramadol, são seguras para contenção química em bugios ruivos (Allouatta guariba clamitans), entretanto, a associação cetamina(S+), midazolam e tramadol apresentou melhor escore de sedação e miorrelaxamento.

\section{REFERÊNCIAS}

Acco A., Pachaly J.R. \& Bacila M. 1999. Síndrome do estresse em animais: revisão. Arqs Ciênc. Vet. Zool. Unipar 2:71-81.

Agoramoorthy G. \& Rudran R. 1994. Field application of Telazol ${ }^{\circledR}$ (tiletamine hydrocloride and zolazepam) to immobilize wild red howler monkeys (Allouata seniculus) in Venezuela. J. Wildl. Dis. 30:417-420.

Ajadi A.R., Olusa T.A., Smith O.F., Ajibola E.S., Adeleye O.E., Adenubi O.T. \&
Makinde F.A. 2009. Tramadol improved the efficacy of ketamine-xylazine anaesthesia in young pigs. Vet. Anaesth. Analg. 36:562-566.

Ayoglu H., Altunkaya H., Bayar A., Turan I.O., Ozer Y. \& Ege A. 2010. The effect of intraarticular combinations of tramadol and ropivacaine with ketamine on postoperative pain after arthroscopic meniscectomy. Arch. Orthop. Trauma Surg. 130:307-312.

Chagas J.A.B., Oleskovicz N., Moraes A.N. de, Flôres F.N., Corrêa A.L., Souza Júnior J.C., Soares A.V. \& Costa A. 2010. Associação de cetamina S(+) e midazolam pelo método convencional de cálculo e pela extrapolação alométrica em bugios-ruivo (Alouatta guariba clamitans): resposta clínica e cardiorrespiratória. Ciência Rural 40:109-114.

Dayer P., Desmeules J. \& Collart L. 1997. Pharmacology of tramadol. Drugs 53:18-24.

Diniz L.S. 1996. Imobilização química em animais silvestres, p.153-163. In: Spinosa H.S., Gorniak S.L. \& Bernardi M. (Eds), Farmacologia Aplicada à Medicina Veterinária. Guanabara Koogan, Rio de Janeiro.

Duque J.C., Oleskovicz N., Guirro E.C.B.P., Valadão C.A.A. \& Soares V.E. 2008. Relative potency of ketamine and S(+)-ketamine in dogs. J. Vet. Pharmacol. Ther. 31:344-348.

Fowler M.E. 1986. Stress, p.24-35. In: Fowler M.E. (Ed.), Zoo and Wild Animal Medicine. $2^{\text {nd }}$ ed. W.B. Saunders Company, Philadelphia.

Friedberg B.L. 1999. The effect of a dissociative dose of ketamine on the bispectral index (BIS) during propofol hypnosis. J. Clin. Anesth. 11:4-7.

Furtado M.M., Nunes A.L.V., Intelizano T.R., Teixeira R.H.F. \& Cortopassi S.R.G. 2010. Comparison of racemic ketamine versus (s+) ketamine when combined with midazolam for anesthesia of Callithrix jacchus and Callithrix penicillata. J. Zoo Wildl. Med. 41:389-394.

Glander K.E., Fedigan L.M., Fedigan L. \& Chapman C. 1991. Capture techniques for three species of monkeys in Costa Rica. Folia Primatol. 57:7082.

Hans P., Dewandre P.Y., Brichant J.F. \& Bonhomme V. 2005. Comparative effects of ketamine on Bispectral Index and spectral entropy of the electroencephalogram under sevoflurane anaesthesia. Brit. J. Anaesth. 94:336-40.

Haskins S.C., Farver T.M. \& Patz J.D. 1985. Ketamine in dogs. Am. J. Vet. Res. 46:1855-1860.

Lauretti G.R., Lima I.C.P.R., Buscatti R.Y. \& Reis M.P. 2000. Avaliação clínica dos efeitos hemodinâmicos, analgésicos, psicodélicos e do bloqueio muscular da cetamina racêmica e de seu S(+) isômero. Revta Bras. Anest. 50:357-362.

Lee C.R., McTavish D. \& Sorkin E.M. 1993. Tramadol: a preliminary review of its pharmacodynamic and pharmacokinetic properties, and therapeutic potential in acute and chronic pain states. Drugs 46:313-340.

Lin H.C., Thurmon J.C., Benson G.J. \& Tranquilli W.J. 1993. Telazol: A review of its pharmacology and use in veterinary medicine. J. Vet. Pharmacol. Ther. 16:383-418.

Lin H.C. 1996. Dissociative anesthetics, p.242-287. In: Thurmon J.C., Tranquilli W.J. \& Benson G.J. (Eds), Lumb and Jones' Veterinary Anesthesia. Lea and Febiger, Baltimore.

Monteiro E.R., Junior A.R., Assis H.M., Campagnol D. \& Quitzan J.G. 2009. Comparative study on the sedative effects of morphine, methadone, butorphanol or tramadol, in combination with acepromazine, in dogs. Vet. Anaesth. Analg. 36:25-33.

Pitt J., Larivière S. \& Messier F. 2006. Efficacy of Zoletil ${ }^{\circledR}$ for field immobilization of raccoons. Wildl. Soc. Bull. 34:1045-1048. 